Hamilton's Principle for a Hydromagnetic Fluid with a Free Boundary

$$
\text { by }
$$

R. I. Dewar

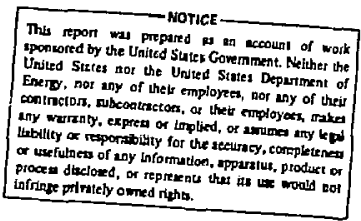

Plasma Physics Laboratory, Princeton University

Prinaeton, New Jersey 08540

\title{
ABST ACT
}

Newcomb's nonlinear fo mulation of Hamilton's principle for an ideal hydromagnetic fluid surrounded by a wall is generalized to allow vacuum and ferromagnetic regions, ane to include the effects of external conductors and circuits. The linear approximation is recovered by expanding to second order in the plasma displacement. When the accumulated charge flowing in each conductor is treated as a generalized variable, the vacuum energy appears as a kinetic energy, with the inductance matrix appearing in the role of a mass tensor. A subsidiary variational principle is used to determine the inductance matrix, thus providing an alternative to the Green's function method. 


\section{INTRODUCTION}

The analytic theory of hydromagnetic stability is largely based on the energy principle of Bernstein, et al., [1] which uses the potential energy functional $\delta \mathrm{W}$. Recently, $[2,3]$ considerable success has also been had in finding the spectrum of small oscillations of a toroidal plasna numerically, using the Rayleigh-Ritz-Galerkin method [4]. These codes also use the potential energy $\delta W$, but combined with the kinetic energy to form the Lagrangian for the system. The Lagrangian formulation is also useful for the general proof of self adjointness of the force operator in $\delta w,[5]$ and for nonlinear problems $[6,7]$.

In practice [2], it has been found inconvenient to use the vector potential form [l] for the perturbed magnetic field $b$ in the vacuum region. Rather, a formulation of $\delta w$ due to Lïst and Martenson [8], based on work by Blank, Friedrichs, and Crad [9] has been used instead. In this formulation, $b$ is represented as the gradient of a single--valued scalar potential $X$, plus subsidiary fields proportioral to the perturbed currents $i_{k}$ in the various conductors. Both $x$ and the $i_{k}$ are constrained to be unique functionals of the displacenent of the plasmavacuum interface, and are typically [2] constructed by Green's function techniques.

A problem with the Green's function method is that the singular nature of the Green's function makes the required 
numerical integracions rather difficult. In this paper we present an alternative, fully variatjonal method, which allows a Galerkin method to be used to calculate the vacuum contributions, thus avoiding the integrals over singular functions encountered in the Green's function method.

A second advantage of the new formulation is that the currents $i_{k}$ are generalized variabies in their own right, and can be constrained or subjected to generalized forces. That is, we can incorporate constraints carsed by the interconnection of the field coils, and include the effect of external circuits. To commence with maximum generality we proceed from Newcomb's fully nonlinear [11] Iagrangian for the ideal hydromagnetic equations, extending it to include a nonconducting region surrounding the plasma. This can include a Eerromaçnetic region, ( $\mu \gg \mu_{0}$ ) but no specific consideration will be given to the effect of such a region and we shall refer to the whole region as the vacuum. We assume the plasma to be topologically toroidal wherever the specific geometry is important. A "general purpose" toroidal configuration is depicted in Fig. 1, which shows a plasma and an arbitrary number of poloidal field coils enclosed within a conducting "wall." To consider the behavior of a plasma in the abserse of a stabilizing shell we can remove three sides of our "wall" to infinity, leaving just: a rod running down the major toroidal axis. Such a corductor is always necessary to maintain the toroidal fielri, so there is always a notional wall present. 
The extension of Newcomb's variational principle [11] is done thisugh two devices. One is the addition of a new kinetic energy term corresponding to the energy in the various electrical circuits contributing to the vacuur field, while the second is the adjoining of a subsidiary variational principle to calculate the inductance matrix as a functional of the positions of the external conductors and of the plasma.

In Section 2 we review the basic equations for the plasma and magnetic field as discussed by previous authors, whereas in Section 3 we discuss the role of the electric fields associated with insulating gaps in the conductors in more detail than has previously been done in the magnetohydrodynamic literature. The electric circuit equations involve an inductance matrix, a fully nonlinear variational principle for which is given in Section 4. In section 5 this principle is used to expand the inductance matrix in powers of the displacement of the plasmavacuum interface. The fully nonlinear Lagrangian including the electrical kinetic energy is presented in Section 6, and expanded in powers of the plasma displacement in section 7 .

The perturbation expansions in sections 5 and 7 are performed using a'novel technique, involving differentiation with respect to the expansion parameter, which allows us to retain familiar Eulerian expressions.

In Section 8 , we indicate how the new methods avoid the singularity problems encountered in the Green's function method. 
Finally, in section 9 we sketch out the types of problems which can be treated using the flexibility inherent in the new approach to the vacuum Lagrangian.

\section{BASIC EQUATIONS (PLASMA AND MAGNETIC)}

The ideal hydromagnetic equations for the mass density $\rho$, pressure $p$, magnetic induction $\underset{\sim}{\mathrm{B}}$, and velocity $\underset{\sim}{\mathrm{V}}$ are $[1,11]$

$$
\frac{d \rho}{d t}=-\rho \nabla \cdot v \sim
$$

$$
\frac{d p}{d t}=-\gamma \underset{\sim}{p} \underset{\sim}{v}
$$

$$
\frac{\mathrm{dB}}{\mathrm{dt}}=\underset{\sim}{\mathrm{B}} \cdot \underset{\sim}{\nabla v}-\underset{\sim}{\mathrm{B}} \nabla \cdot \underset{\sim}{\mathrm{V}}
$$

$$
\rho \frac{d v}{d t}=-\underset{\sim}{\sim}\left(p+\frac{B^{2}}{2 \mu_{o}}\right)+\frac{\underset{B}{B} \cdot \nabla B}{\sim \sim},
$$

where $\mu_{0}$ is the permeability of free space (sI units). We have assumed the gravitational potential to be negligible. These equations apply within the region P occupied by plasma. on the plasma-vacuum interface $S_{p}$, the physical requirement that all. accelerations be finite gives the dynamical 
boundary condition that the component of the stress tensor in the direction normal to $s_{p}$ must be continuous. That is, [1]

$$
\mathrm{B}_{-}^{2} / 2 \mu_{\mathrm{o}}+\mathrm{p}=\mathrm{B}_{+}^{2} / 2 \mu_{\mathrm{O}} ; \underset{\sim}{\mathrm{x} \varepsilon \mathrm{S}_{\mathrm{p}}}
$$

where $B_{-}$and $B_{+}$are respectively the field values measured just inside and just outside the plasma. The magnetic field in the plasma, $\mathrm{B}_{-}$, is assumed to be tangential to the interface initially, and therefore at all later times because of $\mathrm{Eq}$. (3). Because $\underline{n} \cdot \mathbf{B}$ is continuous across any surface, $\underset{\sim}{\mathbf{B}}$ must also be tangential to $s_{p}$. The equation

$$
\underset{\sim}{\nabla} \underset{\sim}{B}=0
$$

applies everywhere. Within the nonconducting region $\mathrm{V}$ surrounding the plasma the low-frequency equation

$$
\underset{\sim}{\nabla} \underset{\sim}{\mathrm{H}}=0 \quad ; \quad \underset{\sim}{\mathrm{x}} \in \mathrm{V},
$$

must also apply, where $\underset{\sim}{\mathrm{H}}$ is the magnetic intensity. Assuming linearity, $\mathrm{B}$ and $\underset{\sim}{\mathrm{H}}$ are related by

$$
\underset{\sim}{\mathrm{B}}=\underset{\sim}{\mathrm{H}},
$$


where $\mu(\underset{\sim}{x})$ is the permeability of the insulating medium. This formulation allows for the possibility of including the effect of an iron-cored transformer in a shell-less tokamak, modeling the iron as a linear magnetic medium with $\mu \gg \mu_{0}$.

In order to determine $\underset{\sim}{D}$ in $V$ we mist also give boundary conditions on the surfaces of any conductors external to the plasma (see Fig. 1). In the spirit of ideal hydromagnetics we take these conductors to be perfectly conducting, so that an appropriate boundary condition on $s_{k}$, the surface of the $k^{\prime}$ th conductor, is

$$
\underset{\sim}{\mathrm{n}} \underset{\sim}{\mathrm{B}}=0 ; \underset{\sim}{\mathrm{x}} \in \mathrm{S}_{\mathrm{k}},
$$

where $\underset{\sim}{n}$ is the unit normal at $\underset{\sim}{\mathrm{x}}$, directed into $\mathrm{V}$. As mentioned previously, Eq. (9) applies also on the plasma surface $(k=p)$, and also on the "wall" ( $k=w)$.

Because $V$ is not a simply connected region $[8,9]$, we must supplement the boundary condition, Eq. (9), with the line integral conditions

$$
\oint_{C_{k}} \underset{\sim}{H} \cdot \underset{\sim}{\ell}=I_{k} ; k=1,2,3, \ldots N+2 \text {, }
$$

(Ampere's law) where $\mathrm{N}$ is the number of conductors external to the plasma, excluding the wall. Thus, $k=N+1$ is 
equivalent to $k=w$ and $k=N+2$ is equivalent to $k=p$. The line integral in Eq. (10) is taken around a path $\mathrm{C}_{\mathrm{k}}$, enclosing only the $k^{\prime}$ th conductor, and running in the right hand sense with respect to the direction in which the current $I_{k}$ in that conductor is measured. The plasma is here regarded as a toroidal conductor; that is, $I_{p}$ is the toroidal plasma current. on the other hand, $I_{w}$ denotes the total poloidal current flowing on the wall. The contour $c_{w}$ for the wall is a loop encircling the major axis between the plasma and the wall. These contours are illustrated in Fig. 1 .

Equations (6) - (10) specify $\underset{\sim}{B}$ throughout $V$, uniquely as a functional of the position of the plasma-vacuum interface $s_{p}$ and the currents $I_{k}$. There is, of course, a net poloidal current in the plasma and also generally a toroidal current on the wall. However, these currents do not contribute to the magnetic field in $V$, so that the total number of contributing currents is $N+2$.

\section{BASIC EQUATIONS (ELECTRICAL)}

In $\mathrm{V}$ the electric field $\underset{\sim}{\mathrm{E}}$ obeys

$$
\underset{\sim}{\nabla} \times \underset{\sim}{E}=-\partial \underset{\sim}{B} / \partial t
$$

on $s_{p}$. Faraday's law may be used to show [1]

$$
\underset{\sim}{\mathbf{n}} \times \underset{\sim}{\mathbf{E}}=\underset{\sim}{\mathbf{n}} \cdot \underset{\sim}{\mathbf{v}_{+}} ; \quad \underset{\sim}{\mathbf{x}} \varepsilon \mathbf{S}_{\mathbf{p}}
$$


while on a fixed surface $S_{k}$ we have

$$
\underset{\sim}{\mathrm{n}} \times \underset{\sim}{\mathrm{E}}=0 ; \underset{\sim}{\mathrm{x}} \in \mathrm{S}_{\mathrm{k}}, \mathrm{k} \neq \mathrm{p}
$$

almost everywhere. However, in order to model the connections to external circuits, we must cut the conductors with thin insulating gaps across which potentials $v_{k}$ (e.m.f.'s) can be applied. The wall may be cut both toroidally and poloidally. We denote the e.m.f. across the toroidal cut by $v_{\theta}$ and that across the poloidal cut by $v_{z}$ (by analogy with $\theta$ and $z$ pinches in cylindrical geometry). We assume the contacts to be arranged so as not to disturb the axisymmetry of the current distribution (if any). If the cuts are represented by the intersections of the $s_{p}$ with the surfaces $\alpha_{k}(\underset{\sim}{x})=0$, such that $\underset{\sim}{n} \cdot \nabla \alpha_{k}=0$, then the generalization of Eq. (13) to include the gaps is

$$
\underset{\sim}{\mathrm{n}} \times \underset{\sim}{\mathbf{E}}=-\mathrm{v}_{\mathrm{k}} \delta\left(\alpha_{\mathrm{k}}\right) \mathrm{n} \times \underset{\sim}{\nabla} \alpha_{\mathrm{k}} ; \underset{\sim}{\mathrm{x}} \varepsilon \mathrm{s}_{\mathbf{k}}, \mathrm{k} \neq \mathrm{p} .
$$

The cuis are illustrated in Fig. 2, and the electric field $\underset{\sim}{E}$ is sketched in Fig. 3. Note that, even though the currents and magnetic field may be axisymmetric, this does not imply that the electric field is axisymmetric. However, we can eliminate the electric field from the problem by integrating Eq. (11) across annular surfaces $\sum_{k}$ bounded by the conductor $k$ and the wall (Fig. 4). There is also a surface $\Sigma_{\theta}$ cutting the vacuum region poloidally. 
Stokes' theorem then reduces the left hand side to a line integral over the contours $\Gamma_{k}$ (Fig. 4). Using Eqs. (12) and (14), we find the set of equations

$$
\begin{aligned}
v_{k}-v_{z} & =\sum_{\ell=1}^{N+2} \frac{d}{d t}\left(I_{k \ell} I_{\ell}\right) ; k \leq N, \\
-v_{z} & =\sum_{\ell=1}^{N+2} \frac{d}{d t}\left(L_{p \ell} I_{\ell}\right) \\
v_{\theta} & =\sum_{\ell=1}^{N+2} \frac{a}{d t}\left(I_{w \ell} I_{\ell}\right)
\end{aligned}
$$

where $I_{k \ell}$ is the mutual inductance between conductor $k$ and conductor $\ell$ (or self-inductarice if $k=\ell$ ). Note that the boundary condition (12) has keen absorbed into the total time derivatives on the right hand sides of Eqs. (16) and (17). Equations (15) - (17) have a very simple interpretation in terms of flux conservation: The flux is frozen-in between the conductors, the plasma, and the wall, except that flux can enter through the cuts, where it flows in or out at a rate equal to the voltage drop across the cut.

The inductance matrix $I_{k \ell}$ an be represented [8] in terms of the magnetic intensity fields $\underline{v}_{k}$ produced by unit currents in each conductor. That is, 


$$
\mathrm{L}_{\mathrm{k} \ell}=\int_{\mathrm{v}} \mu{\underset{\sim}{\mathrm{Y}}}_{\mathrm{k}} \cdot \underset{\sim}{\mathrm{Y}} \mathrm{d} \tau
$$

where $d \tau$ is an element of volume, and $\underset{\sim}{\mathbf{y}_{k}}$ is defined through the equations

$$
\begin{aligned}
& \underset{\sim}{v} \cdot\left(\mu{\underset{\sim}{\mathrm{K}}}_{\mathrm{K}}\right)=0, \quad \underset{\sim}{\mathbf{x}} \varepsilon \mathrm{V} \\
& \underset{\sim}{\nabla} \times \underset{\sim}{\mathbf{Y}_{\mathrm{k}}}=0, \quad \underset{\sim}{\mathbf{x}} \in \mathrm{V} \\
& \underline{n} \cdot{\underset{\sim}{\mathrm{k}}}_{\mathrm{k}}=0, \quad \underset{\sim}{\mathbf{x}} \in S_{\ell}, \text { al. } \ell, \\
& \int_{C_{\ell}} \underset{\sim k}{y_{k}} \cdot d \ell=\delta_{k, \ell}, \text { a11 } \ell .
\end{aligned}
$$

4. VARIATIONAL PRINCIPLE FOR INDUCTANCE MATRIX

One of the purposes of this paper is to formulate the vacuum part of the Lagrangian in a purely variational way. This we do in two steps, the first being to establish a variational principle for the inductance matrix, which, when solved, gives the inductance matrix as a purely geometric functional of the plasma displacement $\underset{\sim}{\xi}$ on the surface of the plasma. This inductance matrix functional appears as one ingredient of Hamilton's principle, which constitutes the second step of the variational formulation. 
This section concerns the first step, which commences with the expression Eg. (1.8) giving $L_{k l}$ as the interaction energy between unit currents in conductors $k$ and $\ell$. We assert that this energy is stationary against arbitrary Jariations $\delta \underset{\sim}{\underset{K}{Y}}$. which obey Eqs, (20) and "(22) as constraints, if and oniy if $\stackrel{Y}{\sim}_{\ell}$ obeys the Eqs. $\backslash(19)$ and (21) (botl for $k=\ell$ and $k \neq \ell$ ). That is, Eqs. (19) and (21) are to follow as the Euler equation and notural boundary condition for the variational principle

$$
\delta I_{\mathbf{k} \ell}=0
$$

To prove that this is true we intruduce a representation for ${\underset{\sim}{\mathrm{K}}}_{\mathrm{k}}$ which satisfies the constraints, Eqs. (20) and (22).

$$
\underset{\sim k}{\mathbf{Y}_{k}}=\underset{\sim \mathrm{k}}{\mathbf{K}_{k}}+\underset{\sim}{\nabla} \mathbf{X}_{\mathbf{k}}
$$

where $\mathrm{K}_{\mathrm{k}}$ is a $\mathrm{f}$ : ed, curl-free field in $\mathrm{V}$, obeying Eq. (22). For example, $\mathbb{K}_{k}$ can be the field of a fixed wirs carrying unit current and entirely enclosed by the surface of conductor $k$. Equation (22) implies that $x_{k}$ is an arbitrary single-valued function. It is now a simple matter to verify that requiring that Eq. (23) be satisfied for arbitrary $\delta \mathrm{x}_{\mathrm{k}}$ leads to Eqs. (19) and (21) as required. Since Eqs. (19) - (22) completely specify the inductance matrix, the variational principle provides a selfcontained procedure for constructing this matrix. 
5. EXPANSION OF INDUCTANCE MATRIX

Supposing that Eqs. (19) - (22) have been solved, the inductance matrix is a functional of the plasma displacement field $\underset{\sim}{\xi}$ at the plasma-vacuum interface. Thus we can expand $L_{k \ell}$ as a functional Taylor series in $\underset{\sim}{\xi}$

$$
\begin{aligned}
& \mathrm{I}_{\mathrm{k} \ell}\left[\xi_{\sim}\right]=\mathrm{L}_{\mathbf{k} \ell}[0]+\int_{\mathrm{s}_{\mathrm{p}}}(0) \mathrm{as} \mathrm{s}^{\prime} \xi_{\sim}^{\prime} \cdot \frac{\Delta \mathrm{L}}{\Delta \xi_{\sim}^{\prime}} \mathrm{k} \ell . \\
& +\frac{1}{2} \int_{S_{p}(0)} d s^{\prime} \int_{S_{p}(0)} d s^{\prime \prime} \xi^{\prime} \cdot \frac{\Delta^{2} L_{k \ell}}{\Delta \xi^{\prime} \Delta \xi^{\prime}}+\xi^{\prime \prime}+\ldots
\end{aligned}
$$

where the use of $\Delta$ rather than the conventional $\delta$ in the functional derivatives is to emphasize that these are all Lagrangian derivatives. That is, $\underline{\sigma}^{\prime}$, etc., are expressed as functions of the unperturbed positions ${\underset{\sim}{\prime}}^{\prime}$, and the surface integrals are over the unperturbed surface $\mathrm{s}_{\mathrm{p}}(0)$.

The functional derivatives can be evaluated with the aid of the variational principle, Eg. (23). To this end we replace $\underset{\sim}{\xi}$ by $\varepsilon \xi$, where $\varepsilon$ is a dummy parameter which will be allowed to run from 0 to 1 . We expand ${\underset{\sim}{\mathrm{k}}}_{\mathrm{k}}$ in powers of $\varepsilon$

$$
\underset{\sim \mathrm{k}}{\mathrm{Y}}=\underset{\sim}{\mathrm{Y}}(0)+\epsilon \underset{\sim}{\nabla \mathrm{X}_{\mathrm{K}}^{(1)}}+(1 / 2) \varepsilon^{2} \underset{\sim}{\nabla} \mathrm{X}_{\mathrm{k}}^{(2)}+\ldots
$$


We also assume that $\underset{\sim}{\xi} \underset{\sim}{x})$ can be amalytically continued into $\mathrm{V}$, so that we can make a change of variable from the "perturbed" position, which we denote by $\mathrm{x}_{\sim}^{*}$, to the "unperturbed" position $\mathbf{x}$. That is, we make the transformation

$$
\left.{\underset{\sim}{x}}^{*}=\underset{\sim}{x}+E \underset{\sim}{\underset{\sim}{x}} \underset{\sim}{\mathbf{x}}, t\right)
$$

to put $L_{k \ell}$ in the form

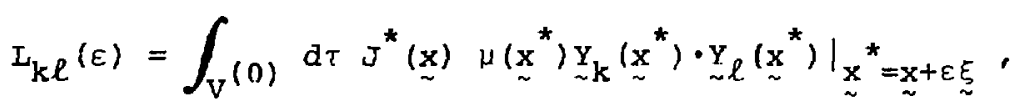

where $\mathrm{J}^{*}$ is the Jacobian of the transformation

$$
\left.J^{\star} \underset{\sim}{x}\right) \equiv \operatorname{det}(\underline{\underline{I}}+\underset{\sim}{\boldsymbol{\Sigma}} \underset{\sim}{\underline{\sim}})
$$

I being the unit dyadic. The advantage of this representation is that the integration is now over the unperturbed region $v^{(0)}$. We now differentiate with respect to $\varepsilon$ to find

$$
\begin{aligned}
& \frac{d L_{k \ell}}{d \varepsilon}=\int_{V}(0) d \tau J^{*}{ }^{*}\left(Y_{\ell}^{*} \cdot \frac{\partial Y_{k}^{*}}{\partial E}+Y_{k^{*}}^{*} \frac{\partial y_{\nu}^{*}}{\partial E}\right) \\
& -\mu_{0} \int_{S}(0) \text { as } J^{*} \underset{\sim}{y_{k}^{*}} \cdot \underset{\sim}{Y_{L}^{*} v^{*}} \cdot \underset{\sim}{\xi}
\end{aligned}
$$


where

$$
{\underset{\sim}{v}}^{*} \equiv(\underline{\underline{I}}+\varepsilon \nabla \underset{\sim}{\xi})^{-1} \cdot \underset{\sim}{n}(0)
$$

$n_{\sim}^{(0)}$ being the unit rormal to $\mathrm{s}_{\mathrm{p}}^{(0)}$, dire ted into $\mathrm{v}^{(0)}$, and the asterisks denote evaluation at $\underset{\sim}{x}=\underset{\sim}{x}+\varepsilon \underset{\sim}{\xi}$ (after any differentiation with respect to $\varepsilon$ ). We have used the following identities to integrate the last term to a surface integral.

$$
\begin{aligned}
& \left(\partial J^{*} / \partial \varepsilon\right)=J^{*}\left(\underline{\underline{I}}+\underset{\sim}{\underset{\sim}{\nabla})^{-1}}: \underset{\sim}{\nabla \xi},\right. \\
& \underset{\sim}{\nabla}\left[\left[J^{*}(\underline{\underline{I}}+\varepsilon \nabla \underset{\sim}{\xi})^{-1 T}\right]=0,\right.
\end{aligned}
$$

where the double dot product is such that $\underset{\sim \sim \sim}{(a b}):(\underset{\sim}{\operatorname{cod}})$ is equal to $(\underset{\sim}{a} \cdot \underset{\sim}{d})(\underset{\sim}{b} \cdot \underset{\sim}{c})$, and superscript $T$ denotes the transposed dyadic. writing

$$
L_{k \ell}(\varepsilon)=L_{k \ell}^{(0)}+\varepsilon L_{k \ell}^{(1)}+(1 / 2) \varepsilon^{2} L_{k \ell}^{(2)}+\ldots \text {, }
$$

expanding the right hand side of Eq. (29) in powers of $\varepsilon$, and equating coefficients we find the first order correction to $\mathrm{L}_{\mathrm{k} \ell}^{(1)}$ from the zeroth order approximation to the right hand side

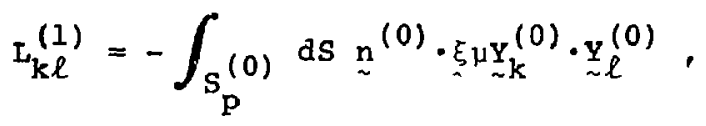


plus a term which vanishes because of the stationarity of $I_{\mathrm{k} \ell}^{(0)}$ with respect to variations of ${\underset{\sim}{k}}_{k}^{(C)}$. Comparing Eqs. (25) and (34) we see that

$$
\Delta \mathbf{L}_{\mathrm{k} \ell} / \Delta \underset{\sim}{\xi}=-\underline{\sim}^{(0)}{ }_{\sim} \mathrm{Y}_{\mathrm{k}}^{(0)} \cdot \underset{\sim}{\mathrm{q}_{\ell}}(0)
$$

As ${\underset{\mathrm{y}}{\mathrm{k}}}_{\mathrm{(})}^{(0)}$ is fixed at this order, $\mathrm{L}_{\mathrm{k} l}^{(1)}$ is trivialiy extremal. To obtain $\mathrm{I}_{\mathrm{k} \ell}^{(2)}$ we need to linearize the right hand side of Eq. (29). It will later be found convenient to tag the small variables with respect to which this linearization is performed with the superscript $\div$. After some integration by parts we find

$$
\begin{aligned}
& I_{k \ell}^{(2)}=\int_{v}(0) d \tau \mu\left[\nabla x_{k}^{(1) \dagger} \cdot \nabla x_{\ell}^{(1)}+\underset{\sim}{(1) \dagger} \cdot \nabla \mathrm{x}_{k}^{(1)}\right]
\end{aligned}
$$

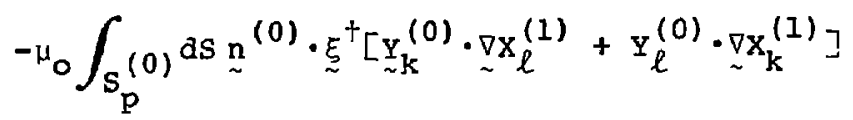

$$
\begin{aligned}
& -\mu_{0} \int_{s_{p}}(0) d s\left[{\underset{\sim}{k}}_{k}^{(0)} \cdot \nabla x_{\ell}^{(1)+}+{\underset{\sim}{y}}_{l}^{(0)} \cdot \underset{\sim}{\nabla x_{k}}(1)\right]_{\sim} \underset{\sim}{(0)} \cdot \underset{\sim}{\xi}
\end{aligned}
$$

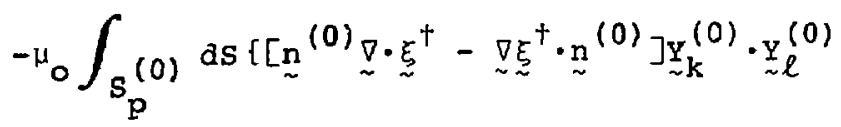

$$
\begin{aligned}
& \left.+\underset{\sim}{\mathbf{n}}{ }^{(0)} \underset{\sim}{\xi^{\dagger}} \cdot \underset{\sim}{\nabla}\left[\underline{Y}_{\mathbf{K}}^{(0)} \cdot{\underset{\sim}{Y}}_{\ell}^{(0)}\right]\right\} \cdot \underset{\sim}{\xi} .
\end{aligned}
$$


Extremizing $\mathrm{L}_{\mathrm{k} \ell}^{(2)}$ with respect to variations in $\mathrm{x}_{\mathrm{k}}^{(1)}$ yields the Euler equation

$$
\underset{\sim}{\nabla} \cdot\left[\underset{\sim}{\nabla x_{\ell}}(1)\right]=0
$$

and the boundary condition on $s_{p}^{(0)}$

$$
\nabla \psi(0) \cdot \nabla \mathrm{x}_{l}^{(1)}={\underset{\sim}{Y}}_{l}^{(0)} \cdot \nabla[\underset{\sim}{\xi} \cdot \nabla \psi(0)]
$$

where $\psi^{(0)}$ is a scalar field with a level surface coinciding with $\mathrm{s}_{\mathrm{p}}^{(0)}$, so that ${\underset{\sim}{\mathrm{n}}}^{(0)}=\underset{\sim}{\nabla}{ }^{(0)} /\left|{ }_{\sim} \psi(0)\right|$. This was introduced to allow integration by parts in the middle term of Eq. (36), using the fact that. $\mathrm{Y}_{\ell}^{(0)}$ is tangential to the surface. We also find the boundary condition on the fixed conductors

$$
\underset{\sim}{n} \underset{\sim}{\nabla x_{l}}(1)=0 ; \underset{\sim}{x \in S_{k}}, k \neq p
$$

Equations (37) and (38) are just the equations normally solved by Green's function techniques, $[2,3,10]$ and the Green's function sclution gives an explicit expression for the second functional derivative when $\mathrm{x}_{k}^{(1)}$ and $\mathrm{x}_{\ell}^{(1)}$ are eliminated in favor of $n \cdot \xi$. However, one of the advantages of the variational formulation of the inductance matrix is that this provides an alternative procedure for its calculation which avoids the use of Green's functions. This wil? be discussed in section 8 . 
The higher order variatic nal derivatives can be evaluated by continuing the expansion in powers of $\varepsilon$ and extremizing order by order, but for linear problems we need go no higher than $o\left(\varepsilon^{2}\right)$.

\section{HAMILTON'S PRINCIPLE}

As the second step in the variational formulation, we seek a Lagrangian $L$ such that Eqs. (4), (5), (15) - (17) follow from the variational principle

$$
\delta \int_{t_{1}}^{t_{2}} 1 d t=0 .
$$

Following Newcomb [11] we integrate Eqs. (1) - (3) so that $\rho, p$, and $\underset{\sim}{B}$ within $P$ are holonomically constrained to vary with the plasma displacement field $\underset{\sim}{\xi}(\underset{\sim}{x}, t)[6]$. In this section we need only the Eulerian variations [11]

$$
\begin{aligned}
& \delta p=-\underset{\sim}{\nabla} \cdot(\rho \delta \xi) \\
& \delta p=-\gamma \underset{\sim}{\nabla} \cdot \delta \underline{\sim}^{\xi}-\delta \underset{\sim}{\xi} \cdot \underset{\sim}{p} \\
& \delta \underset{\sim}{B}=\underset{\sim}{\nabla} \times(\delta \underset{\sim}{\xi} \times \underset{\sim}{B})
\end{aligned}
$$


corresponding to virtual displacements $\delta \underset{\sim}{\xi}$. All quantities in this section are taken to be functions of the perturbed position $\underset{\sim}{\mathbf{x}}={\underset{\sim}{0}}_{0}+\underset{\sim}{\xi}$. We also need the Eulerian variation in the velocity $[11]$

$$
\delta \underset{\sim}{v}=(\partial \delta \underset{\sim}{\xi} / \partial t)+\underset{\sim}{v} \cdot \underset{\sim}{\nabla} \underline{\underline{\xi}}-\delta \underset{\sim}{\xi} \cdot \underset{\sim}{\nabla v}
$$

with Eqs. (40) and (41) it can easily be shown that Eq. (4) follows from the plasma Lagrangian

$$
L_{p}=\int_{v} d \tau\left(\frac{1}{2} \rho v^{2}-\frac{p}{\gamma-1}-\frac{B^{2}}{2 \mu_{0}}\right)
$$

when Hamilton's principle Eq. (39) is invoked for variations $\delta \xi \underset{\sim}{ }$ vanishing on $s_{p}$.

In order to derive the circuit equations (15) - (17) from Hamilton's principle we need the electrical analogue of the position coordinate $x$. As suggested by Maxwell [12], the appropriate generalized coordinate is the charge

$$
a_{k} \equiv \int_{t_{0}}^{t} I_{k} d t
$$

and the corresponding kinetic energy is

$$
T_{\mathrm{e}}=(1 / 2) \sum_{\mathrm{k}, l=1}^{\mathrm{N}+2} \dot{\mathrm{o}}_{\mathrm{k}} L_{\mathrm{k} \ell}\left[\xi_{j}\right] \dot{\phi}_{\ell}
$$


where $\dot{Q}_{k} \equiv I_{k}$ is the analogue of the velocity. Note that $T_{e}$ is the magnetic energy in the insulating region $v$

$$
T_{e}=(1 / 2) \int_{V} \underset{\sim}{H} \cdot \underset{\sim}{B} d \tau
$$

but, because it is a kinetic energy involving the generalized velocities $\dot{Q}_{k}$, it must appear with the opposite sign to the magnetic energy appearirg in $L_{p}$. The circuit equations (15) (17) follow from the Lagrangian for the electricl subsystem

$$
L_{l}=T_{e}+\sum_{k=1}^{N} \Omega_{k}\left(v_{k}-v_{z}\right)-Q_{p} v_{z}+Q_{w} v_{r} .
$$

Here the $V_{k}$ are regarded as exterially imposed e.m.f.'s . It will be shown later how to eliminate the $v_{k}$ by adding on additional contributions to the Lagrangian to represent external circuits.

We now propose that the total Lagrangian of the system is simply the sum of those of the electrical and plasma subsystems

$$
L=L_{e}+L_{p}
$$

The test of this conjecture is to verify that the dynamical boundary condition Eq. (5) is recovered when we allow vixtual

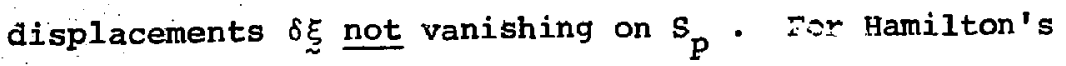


principle to hold under such displacements, the natural boundary condition

$$
n\left(\frac{\mathrm{B}_{-}^{2}}{2 \mu_{0}}+p\right)=-\frac{1}{2} \sum_{\mathrm{k}, \ell=1}^{\mathrm{N}+2} \dot{\mathrm{Q}}_{\mathrm{k}} \frac{\delta \mathrm{I}_{\mathbf{k} \ell}}{\delta \underline{\sim}} \dot{\mathrm{Q}}_{\ell}
$$

must be satisfied. By a method similar to that used in deriving Eq. (35), we can show that the Eulesian functional derivative of $I_{k l}$ is given by

$$
\frac{\delta I_{k \ell}}{\delta \underline{\sim}}=-\underset{\sim}{n} \mu \underset{\sim}{y_{k}} \cdot{ }_{\sim} l
$$

Also, in $\mathrm{V}$, we have

$$
\underset{\sim}{\mathrm{H}}=\sum_{\mathrm{k}=1}^{\mathrm{N}+2} \dot{\underline{Q}}_{\mathrm{k}}{\underset{\sim}{\mathrm{Y}}}_{\mathrm{k}} ; \underset{\sim}{\mathrm{x} \in \mathrm{V}} \text {, }
$$

so that Eq. (48) does indeed reproduce Eq. (5).

\section{EXPANSION OF THE LAGRANGIAN}

Having established the fully nonlinear form of the Lagrangian we must now expand it in powers of the perturbation parameter $E$. The most straightforward method of expansion [11] leads to a form of the potential energy not involving 
gradients of $\mathrm{p}$ or $\mathrm{B}$, similar to that found by Hain, Lüst, and Schlüter [13]. However, it takes a "long and tedious calculation" [11] to prove the equivalence of this form to that of Bernstein, et al., [1]. In this section we show that the expansion method developed in Section 4 leads naturally to the Bernstein et al. form.

To avoid proliferation of superscripts, we shall omit the (0) superscript from the unperturbed quantities. As in Eq. (27), $\mathbf{x}^{*}$ denotes the perturbed position of a fluid element after a displacement $\varepsilon \underset{\sim}{\xi}(x, t)$ from its unperturbed position $\underset{\sim}{x}$.

We must also allow perturbations in the electrical variables [c.f., Eq. (27)]

$$
\begin{aligned}
& Q_{k}^{*}=Q_{k}+\varepsilon q_{k}, \\
& v_{k}^{*}=v_{k}+\varepsilon v_{k} .
\end{aligned}
$$

Using the integral constraint equations [9]

$$
\begin{aligned}
& \rho^{*}=\rho / \mathrm{J}^{*} \\
& \mathbf{P}^{*}=\mathrm{p} / \mathrm{J}^{*} \gamma \\
& {\underset{\sim}{\mathbf{B}}}^{*}=\underset{\sim}{\mathrm{B}} \cdot(\underset{\approx}{\mathrm{I}}+\varepsilon \nabla \underset{\sim}{\xi}) / \mathrm{J}^{*}
\end{aligned}
$$


and the same methods as were used to derive Eq. (29) we find on differentiating Eq. (42) with respect to $E$

$$
\begin{aligned}
\frac{d L_{p}}{d \varepsilon}= & \left.\int_{S_{p}} d S J^{*}\left(\frac{1}{2} \rho^{*} \underset{\sim}{v}+\varepsilon \underset{\sim}{\dot{\xi}}\right)^{2}-\frac{p^{*}}{\gamma-\bar{I}}-\frac{B^{* 2}}{2 \mu_{0}}\right) \nu^{*} \cdot \underset{\sim}{\xi} \\
& +\int_{V} d \tau J^{*} \frac{D}{D \varepsilon}\left(\frac{I}{2} \rho^{*}(\underset{\sim}{v}+\varepsilon \underset{\sim}{\xi})^{2}-\frac{p^{*}}{\gamma-1}-\frac{\tilde{B}^{*}}{2 \mu_{0}}\right)
\end{aligned}
$$

where $\underset{\sim}{\dot{\xi}} \equiv \partial \underset{\sim}{\xi} / \partial t+\underset{\sim}{v} \cdot \underset{\sim}{\nabla} \underset{\sim}{ }$, and the Eulerian derivative $D / D E$ is defined $\mathrm{ky}$

$$
\begin{aligned}
& \frac{D}{D \varepsilon} \equiv \frac{\partial}{\partial \varepsilon}-\underset{\sim}{\xi} \cdot \nabla^{*}, \\
& \left.\nabla^{*} \equiv \underset{\approx}{(I}+\underset{\sim}{\varepsilon \xi}\right)^{-1} \cdot \underset{\sim}{\nabla} .
\end{aligned}
$$

Using the identities

$$
\begin{aligned}
& \frac{\mathrm{DJ}^{*}}{\mathrm{DE}}=\mathrm{J}^{*} \underline{\sim}^{*} \cdot\left(\underline{\xi} / \mathrm{J}^{*}\right), \\
& \frac{\mathrm{D} \rho^{*}}{\mathrm{D} \varepsilon}=-\nabla^{*} \cdot\left(\rho^{*} \xi_{\nu}\right),
\end{aligned}
$$

$$
\frac{D p^{*}}{D \varepsilon}=-\underset{\sim}{\xi} \cdot \underset{\sim}{\nabla} p^{*}-\gamma p^{*} \underset{\sim}{*} \cdot \underset{\sim}{F}
$$


and

$$
\frac{\mathrm{DB}^{*}}{\overline{\mathrm{D}} \varepsilon}={\underset{\sim}{\nabla}}^{*} \times\left(\underset{\sim}{\xi} \times{\underset{\sim}{\mathrm{B}}}^{*}\right),
$$

we can express Eq. (55) in the form

$$
\begin{aligned}
& \frac{d L_{p}}{d \varepsilon}=\int_{S_{p}} d S J^{*}\left[\left(p^{*}+\frac{B^{*} 2}{2 \mu_{0}}\right) \underset{\sim}{v}{ }^{*}+\rho_{\sim}^{*} \underset{\sim}{n} \underset{\sim}{*}\right] \cdot \underset{\sim}{\xi} \\
& +\int_{P} d \tau\left\{\rho v^{*} \cdot \underline{\xi}_{t}-\left[\underset{\sim}{\nabla} \cdot\left(\rho \underset{\sim}{v}{ }^{*}\right)+J^{*} \nabla_{\sim}^{*} P^{*}\right.\right. \\
& \left.\left.-\mu_{0}^{-1} \mathrm{~J}^{*}\left(\underset{\sim}{\nabla} \times \underset{\sim}{\mathrm{B}}{ }^{*}\right) \times \underset{\sim}{\mathrm{B}}{ }^{*}\right] \cdot \xi\right\}
\end{aligned}
$$

where $\underline{\sim}^{\star} \equiv \underset{\sim}{v}+\varepsilon \underset{\sim}{\dot{q}}$, and $\xi_{t}$ denotes $\partial \underset{\sim}{\xi} / \partial t$.

This is a completely general result, ma'iing no assumptions about the uriperturbed background state, which need not even be in equilibrium. For the present purposes we take the unperturbed state to be stationary $(v \equiv 0)$ and in equilibrium

$$
\begin{aligned}
\underset{\sim}{\nabla} \mathrm{P} & =\mu_{0}^{-1}(\underset{\sim}{\nabla} \times \underset{\sim}{\mathrm{B}}) \times \underset{\sim}{\mathrm{B}}, \underset{\sim}{\mathrm{x}} \varepsilon \mathrm{P}, \\
\mathrm{p}+\left(\mathrm{B}_{-}^{2} / 2 \mu_{\mathrm{O}}\right) & =\left(\mathrm{B}_{+}^{2} / 2 \mu_{\mathrm{O}}\right), \underset{\sim}{\mathrm{x} \in S_{\mathrm{p}}}, \\
\mathrm{v}_{\mathrm{k}} & =0, \text { all k. }
\end{aligned}
$$


Differentiating Eq. (46) with respect to $\varepsilon$ we find

$$
\begin{aligned}
\frac{d L}{d E}= & \sum_{k, l=1}^{N+2} \dot{Q}_{k}^{*} L_{k \ell}^{*} \dot{q}_{\ell}+\frac{1}{2} \sum_{k, \ell=1}^{N+2} \dot{Q}_{k}^{*} \frac{d L_{k \ell}^{*}}{d \varepsilon} \dot{Q}_{\ell}^{*} \\
& +\sum_{k=1}^{N} q_{k}\left(v_{k}^{*}-v_{z}^{*}\right)-q_{p} v_{z}^{*}+q_{w} v_{\theta}^{*} \\
& +\sum_{k=1}^{N} Q_{k}^{*}\left(v_{k}-v_{z}\right)-Q_{p}^{*} v_{z}+Q_{w}^{*} v_{\theta} .
\end{aligned}
$$

Here $I_{k \ell}^{*}$ denotes the exact inductance matrix, Eq. (33), because $I_{k l}$ is now being used to denote $I_{k \ell}(0)$. since Eqs. and (38) are here assumed to be solved to give $x_{k}^{(1)}$ as a functional of $\underset{\xi}{ }$ on $\mathrm{s}_{\mathrm{p}}$, we can use integration by parts to cancel the rst two terms of Eq. (36). Using Eq. (50) we have

$\sum_{k, \ell=1}^{N+2} I_{k} L_{k \ell}^{(2)} I_{\ell}=-2 \int_{S_{p}} d \underset{\sim}{B} \cdot \nabla_{X}^{\dagger} \underset{\sim}{n} \cdot \underset{\sim}{\xi}$

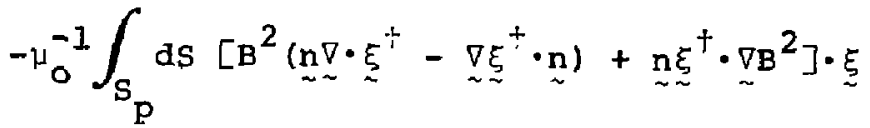


where $x$ is the functional of $\underset{\sim}{\xi}$ defined by

$$
\dot{x} \equiv \sum_{k=1}^{N+2} I_{k} x_{k}^{(I)}
$$

From Eqs. (37) and (38a) we see that $x$ obeys the equations

$$
\begin{aligned}
& \underset{\sim}{\nabla} \cdot(\mu \underset{\sim}{\nabla} X)=0, \underset{\sim}{\mathbf{x}} \varepsilon V, \\
& \left.\underset{\sim}{\nabla} \cdot \underset{\sim}{\nabla X}=\mu_{C}^{-1} \underset{\sim}{B} \cdot \underset{\sim}{\nabla} \underset{\sim}{(\xi} \cdot \nabla \psi\right), \underset{\sim}{x} \in S_{p},
\end{aligned}
$$

and also Eq. (38b).

As in section 5, we need only to linearize the right hand sides of Eqs. (60) and (62) in order to calculate the second order term in the Lagrangian. As the right hand side of $\mathrm{Eq}$. (60) is in Eulerian form, we can recover the usual form of the plasma potential energy with little effort. Using the equilibrium relations Eq. (61) we find the total second order Lagrangian 
$-27-$

$$
\begin{aligned}
& L(2)=\sum_{k, \ell=1}^{N+2} \dot{q}_{k}^{\dagger} L_{k \ell} \dot{q}_{\ell}+\sum_{k=1}^{N}\left[q_{k}^{\dagger}\left(v_{k}-v_{z}\right)+\left(v_{k}^{\dagger}-v_{z}^{\dagger}\right) g_{k}\right] \\
& -q_{p}^{\dagger} v_{z}-v_{z}^{\dagger} q_{p}+q_{w}^{\dagger} v_{\theta}+v_{\theta}^{\dagger} q_{w}
\end{aligned}
$$

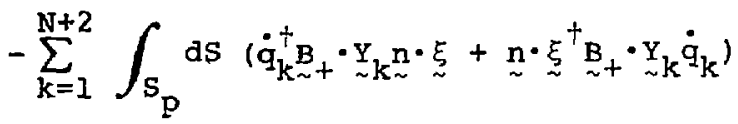

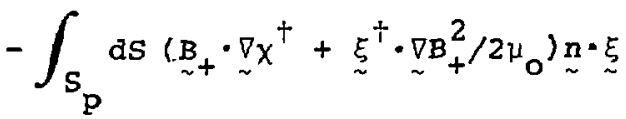

$$
\begin{aligned}
& +\int_{S_{p}} d S\left(-\gamma p \nabla \cdot \underline{\xi}^{\dagger}+\underset{\sim}{B} \cdot{\underset{\sim}{Q}}^{\dagger} / \mu_{0}+\underset{\sim}{\xi^{\dagger}} \cdot \nabla B_{-}^{2} / 2 \mu_{0}\right) \underset{\sim}{n} \cdot \underset{\sim}{\xi} \\
& +\int_{P} d \tau\left\{\rho \xi_{t}^{\dagger} \cdot \xi_{t}-\left[\tilde{\sim}^{\dagger}-\left(\nabla \times \underset{\sim}{Q^{\dagger}}\right) \times \underset{\sim}{B} / \mu_{0}-(\underset{\sim}{\nabla} \times \underset{\sim}{B}) \times \underset{\sim}{Q^{+}} / \mu_{0}\right] \cdot \underset{\sim}{\xi}\right\}
\end{aligned}
$$

where $\tilde{p}$ is the first order Eulerian pressure change

$$
\tilde{p} \equiv-\gamma p \underset{\sim}{\sim} \cdot \underline{\xi}-\underset{\sim}{\xi} \cdot \underset{\sim}{p}
$$

and $\underset{\sim}{Q}$ is the first order Eulerian change in the magnetic field

$$
\underset{\sim}{Q} \equiv \underset{\sim}{\nabla} \times(\underset{\sim}{\xi} \times \underset{\sim}{B})
$$


Because Eq. (67) has been derived by a rigorous expansion method applied to the exact nonlinear Lagrangian, we know that Hamilton's principle must yield the correct linearized equations when $\underset{\sim}{\xi},{\underset{\sim}{\xi}}^{\dagger}, q_{k^{\prime}} q_{k}^{+}$are varied subject to the physical constraints $\xi^{+}=\underset{\sim}{\xi}, q_{k}^{\dagger}=q_{k}$. If, however, we vary $\underset{\sim}{\xi}$ and $q_{k}$ independently of $\underset{\sim}{\xi^{\dagger}}$ and $\mathrm{q}_{\mathrm{k}}^{\dagger}$ and suppose that Hamilton's principle still applies we find the following equations

$$
\begin{aligned}
& \rho{\underset{\sim}{t t}}_{\xi^{\prime}}=-\underset{\sim}{\nabla}+\mu_{0}^{-1}(\underset{\sim}{\nabla} \times \underset{\sim}{Q}) \times \underset{\sim}{\mathrm{B}}+\mu_{0}^{-1}(\underset{\sim}{\nabla} \times \underset{\sim}{\mathrm{B}}) \times \underset{\sim}{Q} \\
& -\gamma P \underset{\sim}{\nabla} \cdot \underset{\sim}{\xi}+\underset{\sim}{B} \cdot Q_{\sim}^{Q}+\underset{\sim}{\xi} \cdot \nabla B_{-}^{2} / 2 \mu_{O} \\
& =\underset{\sim+}{B} \cdot\left(\underset{\sim}{\nabla}+\sum_{k=1}^{N+2} \dot{q}_{k \sim k}{ }_{\sim}\right)+\underset{\sim}{\xi} \cdot \nabla_{B_{+}^{2}}^{2} / 2 \mu_{0}
\end{aligned}
$$

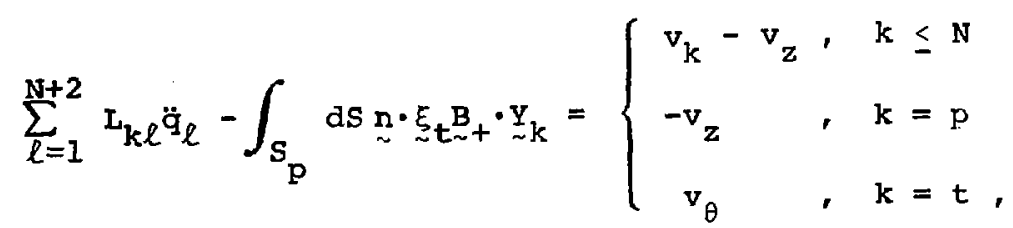

where $\left(\xi_{\sim}^{\dagger}, q^{\dagger}\right)$ has been equated to $(\xi, q)$ only after the variation. 
Equation (70) is obviously correct, being simply the linearized equation of motion. Also, Bernstein et al. [1] showed Eq. (7I) to be the correct linearized dynamical boundary condition, and it can also be shown [8] that Eq. (72) is correct. Thus, it is permissible either to vary $\left(\underset{\sim}{\xi^{\dagger}}, q_{k}^{\dagger}\right)$ and $\left(\xi_{\sim}, q_{k}\right)$ together or to vary $\left(\xi_{\sim}, q_{k}\right)$ independently. These two alternatives are compatible only if we also get the correct equations when we vary $\left(\xi_{\sim}^{\dagger}, q_{k}^{\dagger}\right)$ independently. Thus, the derivation from a Lagrangian constitutes a proof of the selfadjointness of the operator linking $\left(\xi_{\sim}^{\dagger}, q^{\dagger}\right)$ and $(\xi, q)[5]$.

To summarize the complete variational formulation of the linear eigenmode problem, we set forth the three variational problems which must be solved. First, to construct the ${\underset{\sim}{k}}^{\prime} s$ we require that

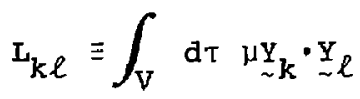

be stationary under variations of the $x_{k}$ defined by the curlfree representation, Eq. (24).

Second, to construct the functional $x[\underset{\sim}{\xi}]$ we require that

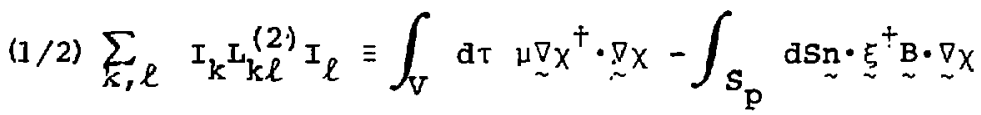

$$
-\int_{S} \text { ds } \underset{\sim}{B} \cdot \underset{\sim}{\nabla} X^{\dagger} \underset{\sim}{n} \cdot \underset{\sim}{\xi}+\text { const. }
$$


be stationary under variations of $x^{+}$or $x$.

Finally, we apply Hamilton's principle: that

$$
\int_{t_{1}}^{t_{2}} L^{(2)} d t
$$

be stationary with respect to variations of ${\underset{\sim}{\xi}}^{\dagger}$ or $\underset{\sim}{\xi}, q_{k}^{\dagger}$ or $\mathrm{q}_{k}$, with $L^{(2)}$ given by Eq. (67).

\section{VACUUM WITHOUT GREEN'S FUNCTIONS}

An immediate benefit of the variational formulation of the vacuum is that it suggests a method for calculating $x$ and the $\mathrm{Y}_{\mathrm{k}}$ 's without encountering the singularity problems inherent in the Green's function method.

We take as a starting point Eqs. (24), (73), and (74). The choice of the current distribution to produce $\underset{\sim \mathrm{k}}{\mathrm{K}}$ is somewhat arbitrary, and should be made in such a way that the field $\mathrm{K}_{\mathrm{k}}$ is approximately tangential to the surface of the conductor $k$. This can be done if necessary by a combination of several line currents. For simplicity, we consider the case $\mu=\mu_{0}$ everywhere in $V$. The choice of $\mathbb{\sim}_{k}$ as a superposition of vacuum H-fields then ensures that the $\mathrm{K}_{\mathrm{k}}$ satisfy $\mathrm{Eg} .(19)$. If we expand $x_{k}$ in a finite set of functions $u_{\alpha}$ obeying Laplace's equation and analytic everywhere in $\mathrm{V}$ and on the surfaces $\mathrm{s}_{\mathrm{k}}$,

$$
x_{k} \underset{\sim}{(x)}=\sum_{\alpha} x_{k}^{\alpha} u_{\alpha}
$$


we can automatically satisfy Eq. (19). The boundary conditions Eqs. (21) and (22) remain to be approximated variationally. A suitable choice for the $u_{\alpha}$ would be toroidal harmonics with toraidal axes coinciding with the line currents used to produce the $K_{k}{ }^{\prime} s$.

Inserting the Ansatz, Eq. (76) into Eq. (73) and integrating by parts we find

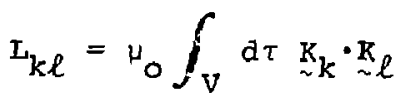

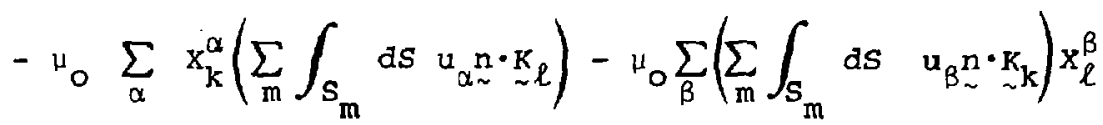

$$
\begin{aligned}
& +\mu_{\circ} \sum_{\alpha, \beta} x_{k}^{\alpha} A_{\alpha \beta} x_{\ell}^{\beta}
\end{aligned}
$$

where $\sum_{m}$ sums over all $N+2$ surfaces, and $A_{\alpha \beta}$ is the matrix

$$
A_{\alpha \beta} \equiv-\sum_{\mathbb{m}} \int_{s_{m}} \text { ds } u_{\alpha \sim} n \cdot \nabla u_{\beta} .
$$

Requiring that $L_{k \ell}$ be stationary with respect to arbitrary variations in the coefficients $x_{k}^{\alpha}$ yields the equation 


$$
\sum_{\beta} A_{\alpha \beta} x_{\ell}^{\beta}=\sum_{m} \int_{s_{m}} \text { ds } u_{\alpha \sim} n \cdot k_{\ell} .
$$

Inversion of $A_{\alpha \beta}$ yields the required coefficients $x_{\ell}^{\beta}$, from which can be constructed the ${\underset{\sim}{\mathrm{k}}}_{\mathrm{k}}{ }^{\prime} s$ and $\mathrm{I}_{\mathrm{k} \ell}$.

Similarly, to construct an approximate solution for $X$, we expand $\chi$ in the same representation

$$
x=\sum_{\alpha} x_{\alpha} u_{\alpha}
$$

insert this in Eq. (74), and integrate by parts to find

$$
\begin{aligned}
& (1 / 2) \sum_{k, l} I_{k} I_{k l}^{(2)} I_{l}=\mu_{0} \sum_{\alpha, \beta} x_{\alpha}^{\dagger} A_{\alpha \beta} x_{\beta} \\
& -\sum_{\beta} x_{B} \int_{S_{p}} d s \underset{\sim}{n} \cdot \underset{\sim}{\xi_{B}^{+}} \underset{\sim}{\sim} \underset{\sim}{\nabla} u_{B} \\
& -\sum_{\alpha} \chi_{\alpha}^{\dagger} \int_{S_{p}} d s \underset{\sim}{z} \cdot \underset{\sim}{\xi_{\alpha}} \cdot \underline{\sim}_{\alpha}+\text { const. }
\end{aligned}
$$

variation of $x_{\alpha}^{\dagger}$ yields the equation 


$$
\mu_{0} \sum_{B} A_{\alpha \beta} x_{B}=\int_{S_{p}} d s \underset{\sim}{n} \cdot \underset{\sim}{\xi B} \cdot \underset{\sim}{\nabla} u_{\alpha} .
$$

Thus to solve for the coefficients $\chi_{\beta}$ we need only the inverse of $A_{\alpha \beta}$, which must already have been calculated to obtain $L_{k l}$.

\section{CIRCUIT EQUATIONS}

In this section we sketch out the types of problems which can be treated using the freedom inherent in our formulation.

First, we suppose all the insulating gaps to be shorted out, we can set ail the $v_{k}$ to zero. Then $q_{k}$ does not appear in the Lagrangian and we can use the currents $i_{k} \equiv \dot{q}_{k}$ as generalized coordinates. The Euler equation corresponding to variation of $i_{k}$ is the time integrated form of Eq. (72) with the right hand side set to zero. Thus, by inverting the inductance matrix, we can find the $i_{k}$ 's as functionals of $\underset{\sim}{\xi}$ and eliminate them from the Lagrangian. Using the identity

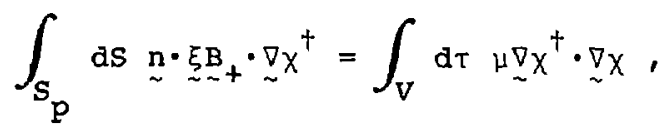

we can then put Eq. (67) in the form

$$
L^{(2)}=\int_{\mathrm{P}} d \tau \rho \underset{\sim}{t} \cdot \underset{\sim}{\dagger}-2 \delta w .
$$


where $\delta \mathrm{W}$ is the potential energy functional found by Bernstein et al. [1], with the vacuum energy in the scalar potential form [8]

$$
\begin{aligned}
& 2 \delta W=\int_{V} \mu\left(\underset{\sim}{\nabla} X^{\dagger} \cdot \underset{\sim}{\nabla} X+\sum_{k, \ell=1}^{N+2}\left(i_{k \sim k}^{+} \underset{\sim}{Y}\right) \cdot\left(i_{\ell \sim \ell}^{\underset{Y}{Y} \ell}\right)\right) \\
& +\int_{S_{p}} d S \underset{\sim}{\xi^{\dagger}} \cdot \underset{\sim}{\nabla}\left(\frac{B_{+}^{2}}{2 \mu_{0}}-\frac{B_{-}^{2}}{2 \mu_{o}}-p\right) \underset{\sim}{n} \cdot \underset{\sim}{\xi}
\end{aligned}
$$

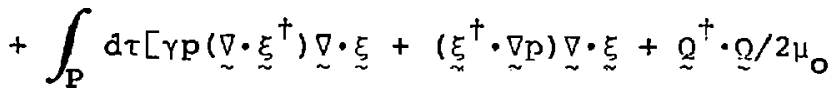

$$
\begin{aligned}
& \left.-(\underset{\sim}{\nabla} \times \underset{\sim}{B}) \times \underset{\sim}{Q}{ }^{\dagger} \cdot \underset{\sim}{\xi} / \mu_{0}\right] .
\end{aligned}
$$

Thus we have proved that the previous forms of the variational principle can be recovered as a special case of the present one. It is now of interest to explore the types of problems which can be attacked using the extra freedom obtained by treating the currents or charges as generalized coordinates. 
The first problem which can be treated is the effect of passive feedback on stability. Suppose, for instance, that all gaps are shorted out except for two ( $k=1$ and 2, say), which are instead connected so that the conductors are in series (see Fig. 5). Then

$$
\mathrm{v}_{1}=\mathrm{v}_{2}=\mathrm{v}, \text { say, }
$$

and we also have the charge conservation constraint

$$
q_{1}=-q_{2}
$$

Inspecting Eq. (67) we see that $v$ acts as a Iagrange multiplier with respect to the constraint Eq. (87). That is, by adjoining Eq. (87) to F.q. (72) we can solve for $\mathrm{v}$ and again eliminate all the currents $i_{k} \equiv \dot{q}_{k}$ as functionals of $\underset{\xi}{\xi}$. In fact Eq. (85) is unchanged, except for the implicit functional dependence of the $i_{k}$ on $\underline{\xi}$.

Now suppose that all gaps are shorted, except for $k=1$, which is connected to an external circuit with inductance $L$. We also attempt to short the gap to high frequency currents by connecting a capacitor $C$ across it, as depicted in Fig. 6. This problem can be treated by adding a contribution to the Lagrangian corresponding to the two circuits being driven by the potential $v_{1}$. The Lagrangian for the capacitor circuit $c$ 
is a potential energy term plus an interaction term to represent the effect of the potential $v_{1}$

$$
L_{c}^{(2)}=-q^{\dagger} q / c-q^{\dagger} v_{1}-q v_{1}^{\dagger}
$$

while that for the inductor circuit i is a kinetic energy term plus an interaction term

$$
L_{i}^{(2)}=\left(\dot{q}_{1}^{\dagger}-\dot{q}^{\dagger}\right) L\left(\dot{q}_{1}-\dot{q}\right)-\left(q_{1}^{\dagger}-q^{\dagger}\right) v_{1}-\left(q_{1}-q\right) v_{1}^{\dagger},
$$

where we have built in the charge conservation constraint by expressing the charge in the inductor circuit in terms of $q_{1}$ and $q$, the charge in the capacitor circuit.

When Eqs. (67), (88), and (89) are added, the terms involving $v_{1}$ cancel, as they must because there is no excitation external to the total system. However, the presence of the term $\mathrm{q}^{\dagger} \mathrm{q} / \mathrm{C}$ represents a qualitative change in the nature of the Lagrangian since $\dot{q}$ cannot be treated as a generalized coordinate. If we assume the time dependence $\exp (-i \omega t)$ and work in terms of the $i_{k}$ variables and $q, L_{i}^{(2)}$ will contain terms linear in $w$. If we retain the $q_{k}$ variables, $L^{(2)}$ now will contain linear terms in $\omega$. Either way the problem is no longer a standard eigenvalue problem for $\omega^{2}$, and will require new solution techniques.

Finally, we remark that if we apply a true external potential $v_{1} \exp (i \omega t)$, the finite element method can still be used to 
calculate the open loop response $q_{1}\left(v_{1}, w\right)$. Thus, by using the Nyquist technique it should be possible to examine the effect of an active feedback circuit on stability and growth rates.

\section{CONCLUSION}

In the foregoing sections we have done several things:

(i) We have generalized Newcomb's [11] nonlinear Lagrangian by the inclusion of a vacuum region. The availability of a nonlinear Lagrangian offers not only the possibility of investigating mode coupling effects $[7]$, but also the possibility of making a linear stability code self-correcting with respect to errors in the approximation to the equilibrium whose stability is to be investigated.

(ii) We have found a method of expansion for the Lagrangian which needs only a linearization to treat linear problems and which produces the familiar form of the potential energy in a natural manner.

(iii) We have found variational nrinciples for the inductance matrix $L_{k \ell}$ and scalar potential $x$ which allows Galerkin's method to be used in the vacuum region.

(iv) We have introduced generalized coordinates to describe the currents flowing in the plasma and external conductors, thus allowing the treatment of external circuits. 


\section{ACKNOWLEDGMENTS}

It is a pleasure to acknowledge useful conversations with Drs. M. S. Chance, R. M. Kulsrud, J. L. Johnson, J. M. Greene, and R. C. Grimm.

This work was supported by United States Department of Energy Contract No. EY-76-C-02-3073. 


\section{REFERENCES}

[1] BERNSTEIN, I. B., FRIEMAN, E. A., KRUSKAL, M. D., and KULSRUd, R. M., Pros. Roy. Soc. (London) 244A, 17 (1958).

[2] GRIMM, R. C., GREENE, J. M., and JOHNSON, J. I., , in Methods in Computational Physics, V. 16, ed. J. Killeen (Acadenic Press, New York, 1976), p. 253.

[3] BERGER, D., Numerical Computations of the Ideal Magnetohydrodysamic Stability of Small Aspect Ratio Tokamaks (Ph.D. Thesis, Ecole Polytechnique Federal de Lausanne, 1977).

[4] STRANG, G. and FIX, G. J., An Analysis of the Finite Elerent Method (Prentice Hall, Englewood Cliffs, New Jersey, 1973).

[5] KULSRUD, R. M., Astrophys. J. 152, 1121 (1968).

[6] DEWAR, R. Lo, Phys. Fluids 13, 2710 (1970).

[7] DAGAZIAN, R. Y., Nucl. Fusion 16, 12 (1976).

[8] LÜsT, R. and MARTENSON, E., Z, Naturforsch. 15a, 706 (1960).

[9] BLANK, A. A., FRIEDRICHS, R. O., and GRAD, H., Theory of Maxwel1's Equations Without Displacement Current, Notes on Magneto-rydrodynamics $V$, Report NYO-6486, Courant Institute of Mathematical Sciences, New York University (1957).

[10] MARDER, B. M., Phys. Fluids 17, 634 (1974).

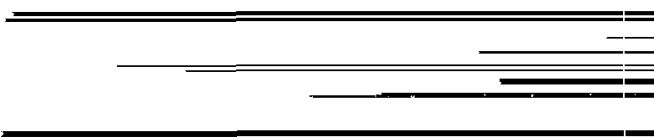


[11] NEWCOMB, W. A., Nucl. Fusion Supp1. Pt. 2, 451 (1962).

[12] MAXWELL, J. C., A Treatise on Electricity and Magnetism (Dover, New York, 1954), 3rd ed., v. 2, p. 223.

[13] HAIN, K., LÜST, R., and SCHLÜTER, A., z. Naturforsch. $12 a, 833$ (1957). 


\section{FIGURE CAPTIONS}

Fig. 1. General toroidal configuration: plasma, poloidal conductors, and wall. Three sides of the wall may go to infinity leaving a conductor on the major axis to give the toroidal field. The currents $I_{k}$ and Ampere contours $c_{k}$ are also shown.

Fig. 2. Insulating gaps defined by the surfaces $\alpha_{k}=0$, across which e.m.f.'s $v_{k}$ are applied.

Fig. 3. Close-up of electric field in the vicinity of an insulating gap, showing how the tangential component is effectively a $\delta$ function.

Fig. 4. Surfaces $\sum_{k}$ and contours $\Gamma_{k}$ used for integrating Faraday's equations.

Fig. 5. Two conductors in series, showing how Kirchhoff's laws give constraints on the circuits.

Fig. 6. Addition of external circuits to the system. 


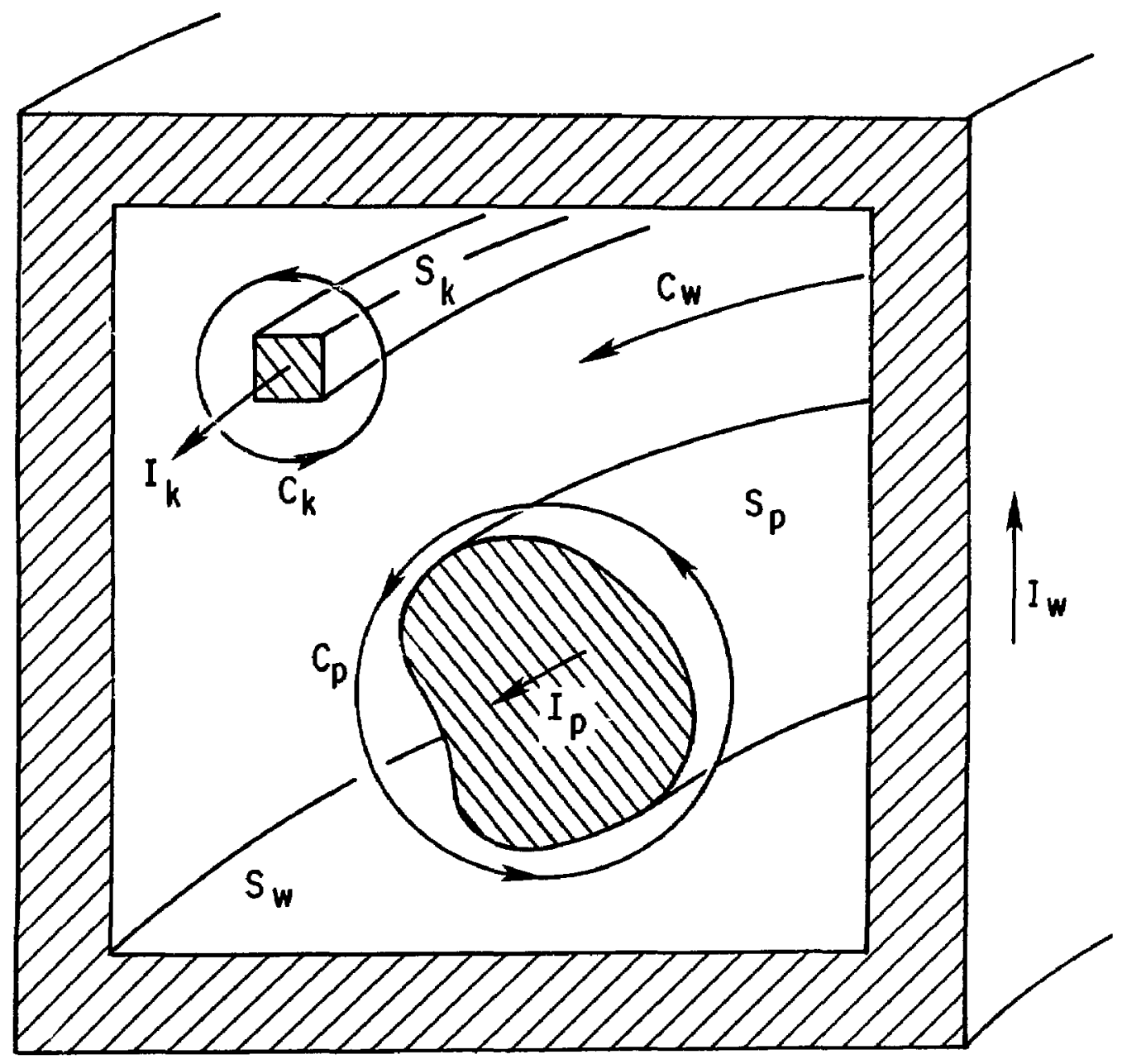

Fig. 1. 782019 


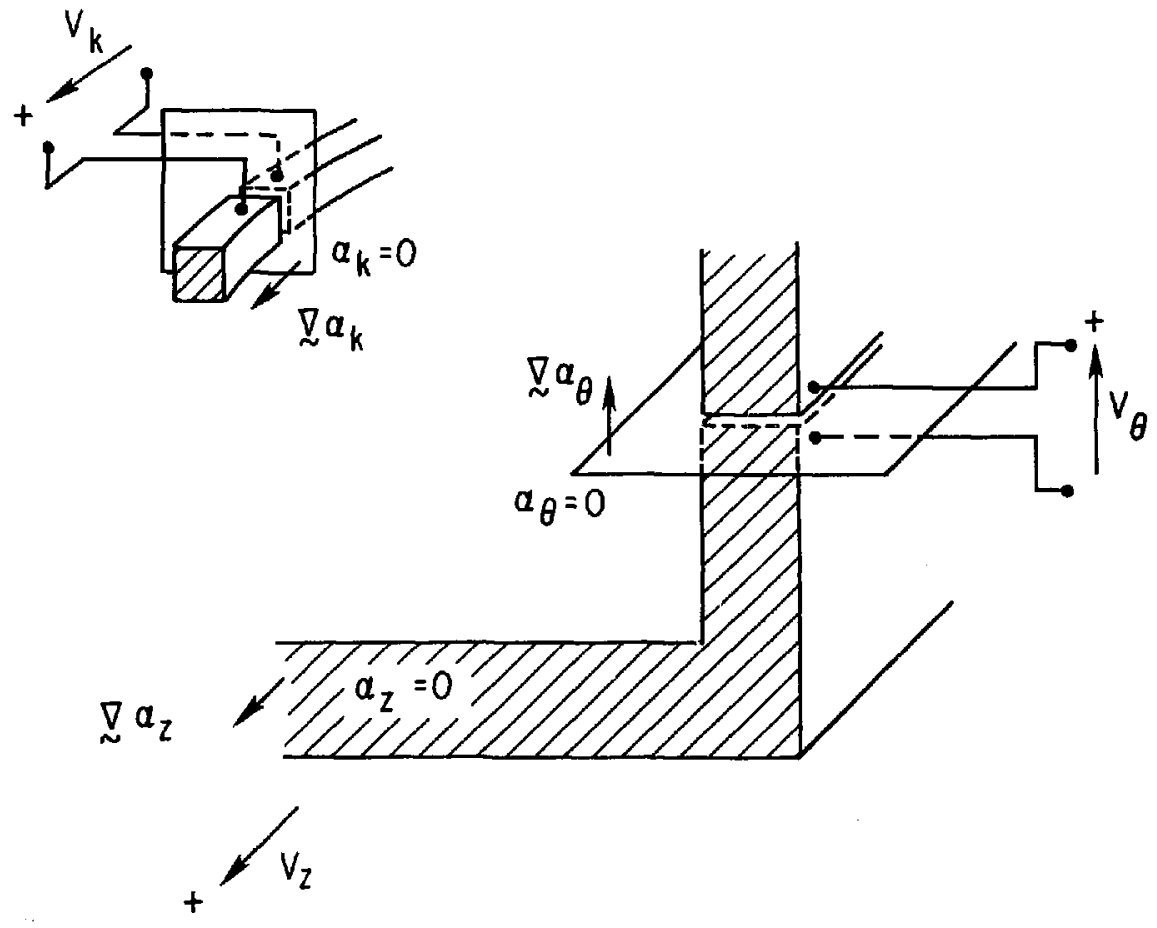

Fig. 2. 782021 


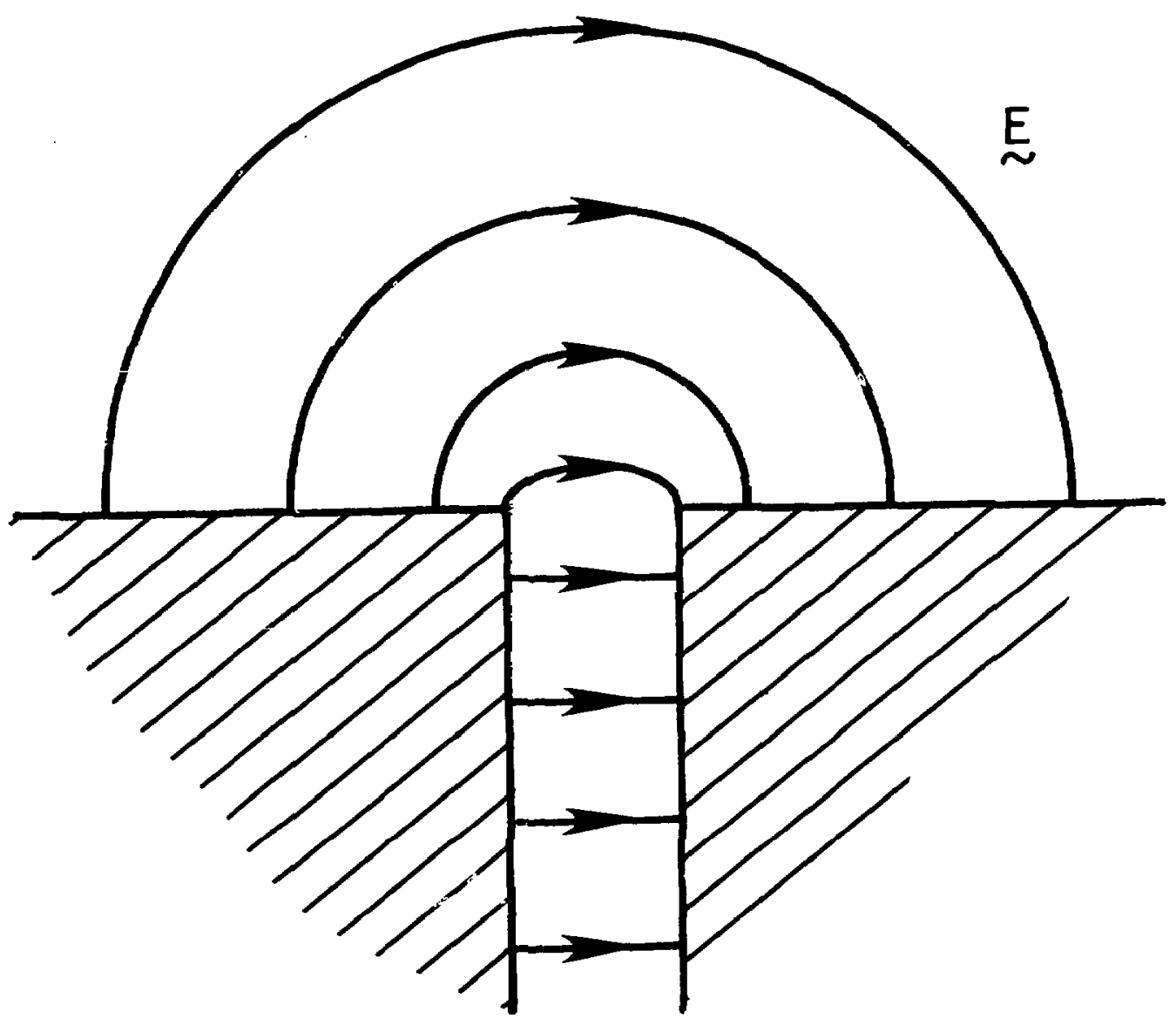

Fig. 3. 782018 

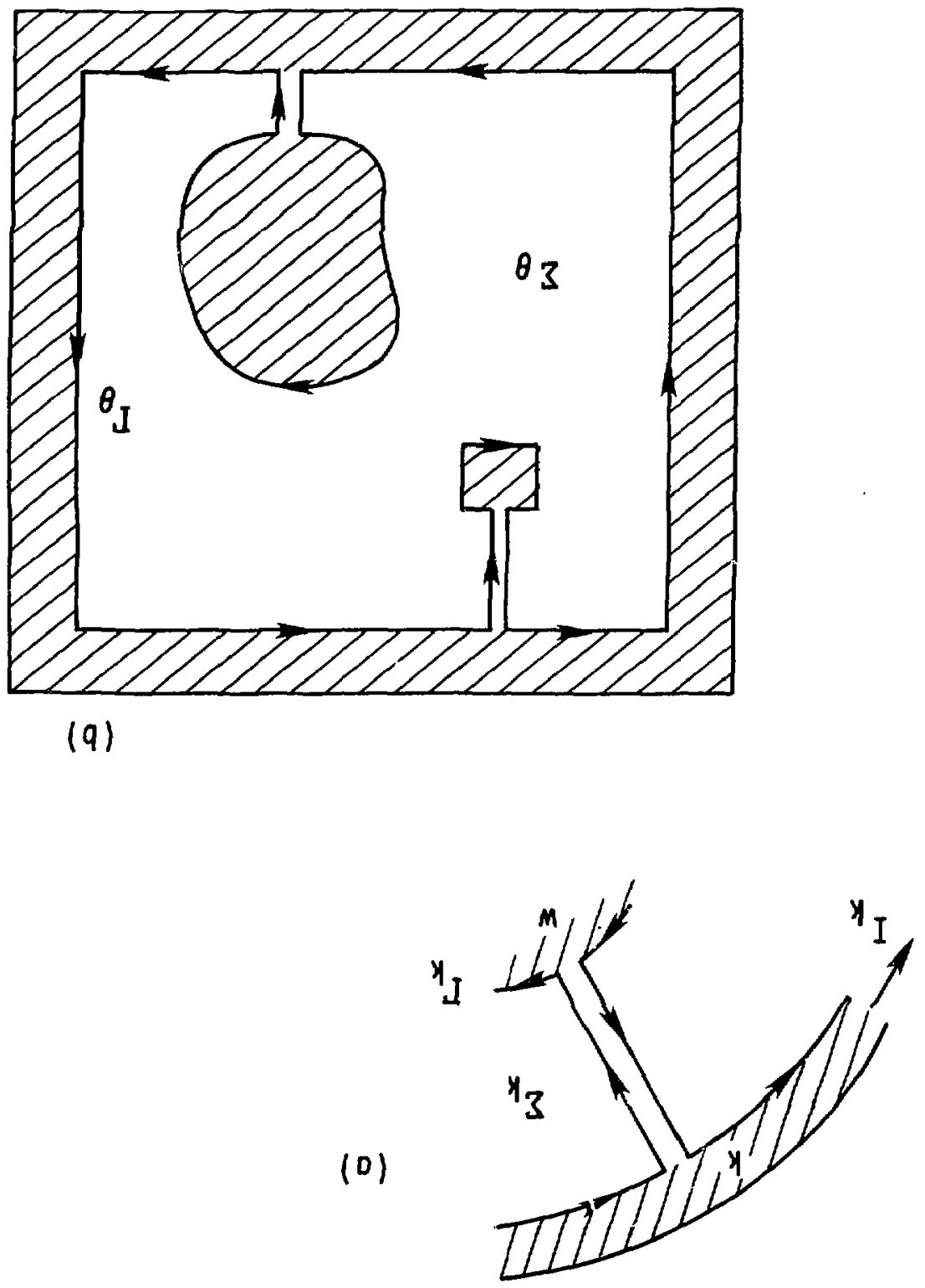

Fig. 4. 782020 


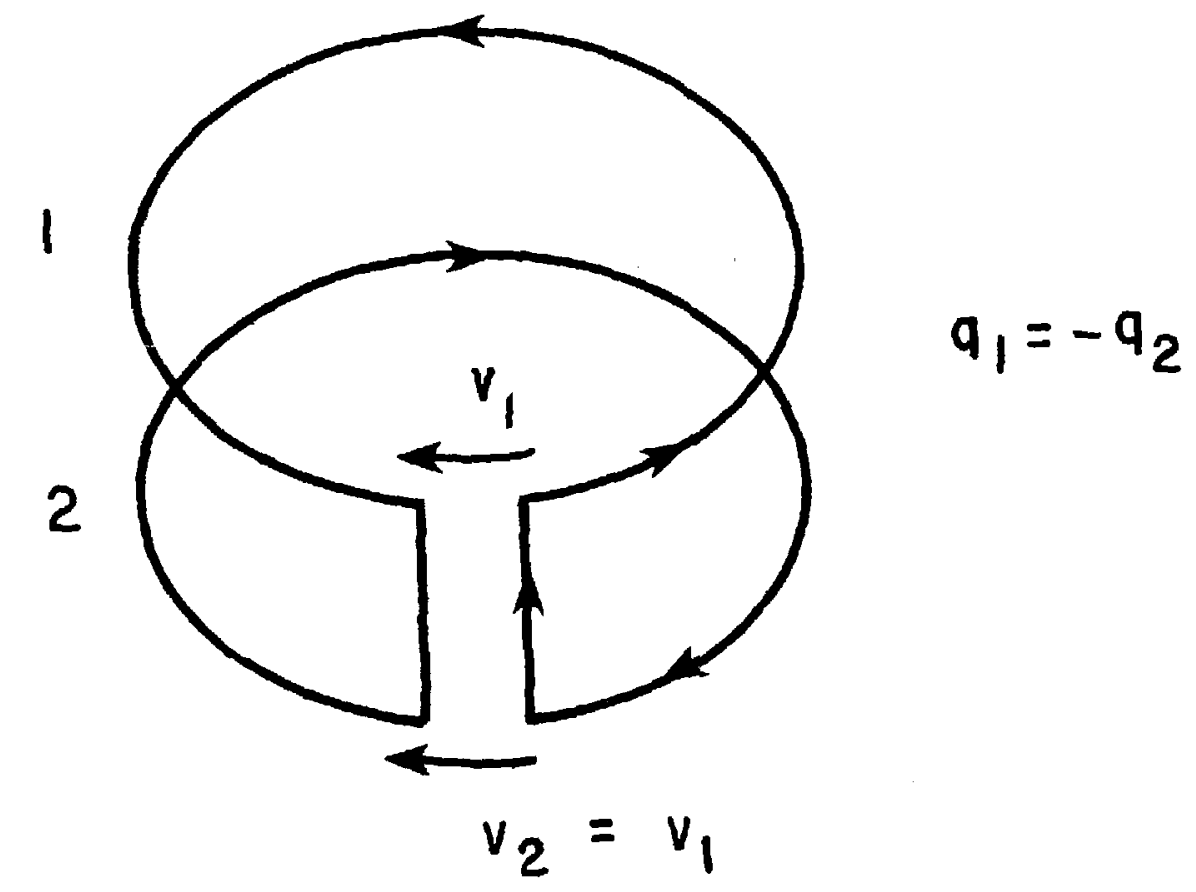




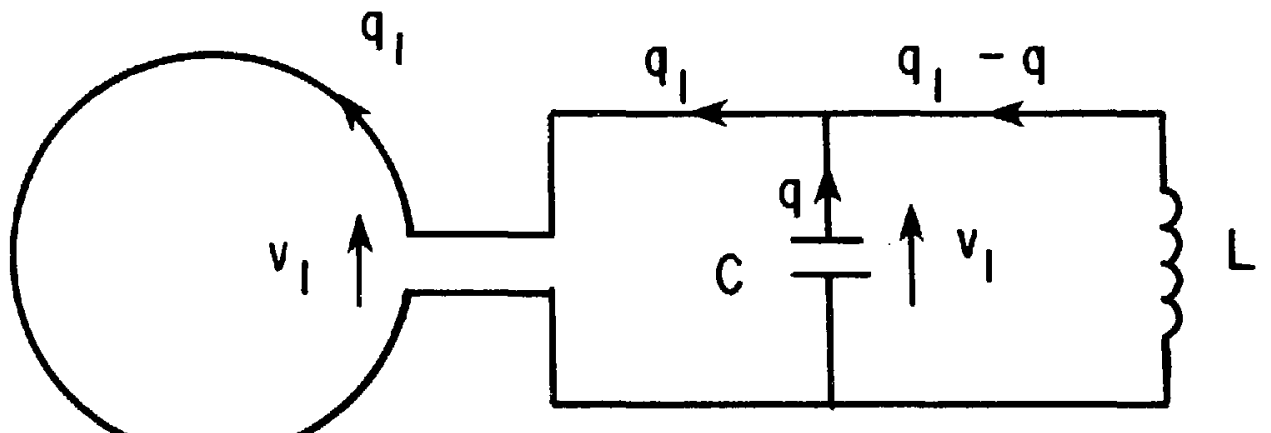

$\frac{1}{1}$

Fig. 6. 782016 